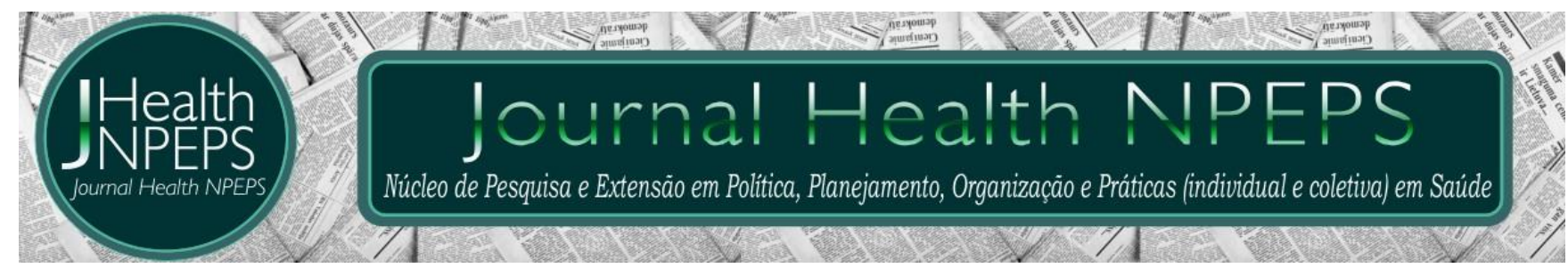

http://dx.doi.org/10.30681/252610104296

ARTIGO ORIGINAL

\title{
Perspectivas de familiares de crianças e adolescentes em tratamento oncológico quanto à assistência multiprofissional
}

\section{Perspectives of family members of children and adolescents undergoing cancer treatment regarding multidisciplinary care}

\section{Perspectivas de familia de niños y adolescentes en tratamiento oncológico cuanto a la atención multidisciplinaria}

\section{Patrick Leonardo Nogueira da Silva ${ }^{1}$, Fabiana Gomes Santos Martins², Josiana Damasceno Freire ${ }^{3}$, Fábio Batista Miranda ${ }^{4}$, Ana Augusta Maciel de Souza ${ }^{5}$}

\begin{abstract}
RESUMO
Objetivo: identificar as perspectivas de familiares de crianças e adolescentes com câncer quanto à assistência prestada pela equipe multiprofissional. Método: trata-se de estudo descritivo, exploratório e com abordagem qualitativa, realizado com familiares acompanhados por instituições hospitalares de Minas Gerais. Utilizou-se entrevista semiestruturada, executada por meio da aplicação de um formulário contendo 11 questões norteadoras, e os depoimentos gravados por meio de um celular com gravador MP3. Em seguida, foram transcritos na íntegra, categorizados e discutidos por meio de Análise de Conteúdo. Resultados: observou-se a participação ativa da equipe multiprofissional na prestação de assistência ao paciente oncológico e sua família. Conforme os depoimentos, a
\end{abstract}

\footnotetext{
${ }^{1}$ Enfermeiro. Mestrando pelo Programa de Pós-Graduação em Cuidado Primário em Saúde da Universidade Estadual de Montes Claros (PPGCPS/UNIMONTES). Professor da Escola Técnica de Saúde do Centro de Educação Profissional e Tecnológica (ETS/CEPT) da UNIMONTES. Montes Claros, Minas Gerais, Brasil. E-mail: patrick mocesp70@hotmail.com ORCID ID: http://orcid.org/0000-0003-2399-9526 Autor para correspondência - Endereço: Universidade Estadual de Montes Claros (UNIMONTES), Centro de Ciências Biológicas e da Saúde (CCBS), Departamento de Enfermagem - Avenida Professor Rui Braga, s/no, Prédio 06, Sala 104, Vila Mauriceia, Montes Claros, Minas Gerais, Brasil. CEP: 39.401-089.

${ }^{2}$ Enfermeira. Graduada em Enfermagem pelas Faculdades Integradas Pitágoras de Montes Claros (FIPMoc). Montes Claros, Minas Gerais, Brasil. E-mail: fabi2881@hotmail.com ORCID ID: http://orcid.org/0000-0002-0645-6151

${ }^{3}$ Enfermeira. Graduada em Enfermagem pelas Faculdades Integradas Pitágoras de Montes Claros (FIPMoc). Montes Claros, Minas Gerais, Brasil. E-mail: josianedamasceno@hotmail.com ORCID ID: https://orcid.org/0000-0002-6786-8221

${ }^{4}$ Enfermeiro. Doutorando pelo Programa de Pós-Graduação em Enfermagem e Biociências da Universidade Federal do Estado do Rio de Janeiro (PPGENFBIO/UNIRIO). Rio de Janeiro, Rio de Janeiro, Brasil. E-mail: fbtmiranda@gmail.com ORCID ID: http://orcid.org/0000-0003-0934-980X

${ }^{5}$ Enfermeira. Mestre em Ciências. Professora do Departamento de Enfermagem das Faculdades Integradas Pitágoras de Montes Claros (FIPMoc) e da Universidade Estadual de Montes Claros (UNIMONTES). Montes Claros, Minas Gerais, Brasil. E-mail: anamaciel@uai.com.br ORCID ID: https://orcid.org/0000-0003-4755-3297
}

Este artigo está licenciado sob forma de uma licença Creative Commons Atribuição 4.0 Internacional, que permite uso irrestrito, distribuição e reprodução em qualquer meio, desde que a publicação original seja corretamente citada 
relação médico-paciente é permeada pela confiança, o que favorece o vínculo entre ambos e repercute no processo terapêutico. Quanto ao atendimento de enfermagem, psicologia, nutrição e serviço social, atribui-se a preocupação contínua com o diálogo, as necessidades específicas de cada paciente e orientações para realização dos procedimentos e acompanhamentos posteriores. Considerações finais: a assistência da equipe multiprofissional junto à criança e adolescente com câncer é vista como benéfica e deve ser integralmente compartilhada entre todos os envolvidos no processo de cuidado.

Descritores: Neoplasias; Criança; Adolescente; Família; Equipe de Assistência ao Paciente.

\section{ABSTRACT}

Objective: to identify the perspectives of family members of children and adolescents with cancer regarding the assistance provided by the multiprofessional team. Method: it is a descriptive, exploratory study with a qualitative approach, carried out with family members accompanied by hospitals in Minas Gerais. A semi-structured interview was used, carried out by applying a form containing 11 guiding questions, and the testimonies recorded using a cell phone with MP3 recorder. Then, they were transcribed in full, categorized and discussed through Content Analysis. Results: it was observed the active participation of the multiprofessional team in providing assistance to cancer patients and their families. According to the testimonies, the doctor-patient relationship is permeated by trust, which favors the bond between both and affects the therapeutic process. As for nursing care, psychology, nutrition and social work, the ongoing concern with dialogue, the specific needs of each patient and guidelines for carrying out the procedures and subsequent follow-up are attributed. Final considerations: the assistance of the multidisciplinary team with children and adolescents with cancer is seen as beneficial and must be fully shared among all those involved in the care process.

Descriptors: Neoplasms; Child; Adolescent; Family; Patient Care Team.

\section{RESUMEN}

Objetivo: identificar las perspectivas de los familiares de niños y adolescentes con cáncer con respecto a la asistencia brindada por el equipo multiprofesional. Método: es un estudio exploratorio descriptivo con enfoque cualitativo, realizado con familiares acompañados por hospitales en Minas Gerais. Se utilizó una entrevista semiestructurada, realizada mediante la aplicación de un formulario que contiene 11 preguntas orientadoras, y los testimonios grabados utilizando un teléfono celular con grabadora de MP3. Luego, se transcribieron en su totalidad, se clasificaron y se discutieron a través del Análisis de contenido. Resultados: se observó la participación activa del equipo multiprofesional en la prestación de asistencia a pacientes con cáncer y sus familias. Según los testimonios, la relación médico-paciente está impregnada de confianza, lo que favorece el vínculo entre ambos y afecta el proceso terapéutico. En cuanto a la atención de enfermería, psicología, nutrición y trabajo social, se atribuye la preocupación constante por el diálogo, las necesidades específicas de cada paciente y las pautas para llevar a cabo los procedimientos y el seguimiento posterior. Consideraciones finales: la asistencia del equipo multidisciplinario con niños y adolescentes con cáncer se considera beneficiosa y debe compartirse plenamente entre todos los involucrados en el proceso de cuidado.

Descriptores: Neoplasias; Niño; Adolescente; Familia; Grupo de Atención al Paciente. 


\section{INTRODUÇÃO}

Na infância, o câncer se relaciona a um grupo de mais de 200 doenças de etiologia multifatorial, particularmente associada à alteração no processo de divisão celular e multiplicação desordenada de células ${ }^{1}$. 0 câncer em crianças e adolescentes compreende aproximadamente $1 \%$ de todas as neoplasias malignas. Os tipos de cânceres mais frequentes em crianças do mundo equivalem ao grupo das leucemias, linfomas e dos tumores do sistema nervoso central (SNC) $)^{2}$.

Embora o conhecimento sobre o câncer tenha evoluído em relação aos avanços tecnológicos no tratamento da doença, o seu diagnóstico gera uma sucessão de impactos, obrigando, na maioria dos casos, as famílias a tomarem decisões e assumirem novas responsabilidades, mesmo não estando preparadas para tais desafios ${ }^{3}$.

Quanto ao tratamento, diferentes situações interferem nos papéis da criança com diagnóstico oncológico, sendo fundamental o acolhimento da família e profissionais, para amenizar os efeitos negativos dessa etapa. 0 ambiente de cuidado ofertado deve ser acolhedor para ambos, a fim de proporcionar adesão ao tratamento e mais confiança na equipe e nos procedimentos que serão realizados ${ }^{4}$.

Frente a isso, os impactos da doença precisam ser compreendidos levando em consideração as condições emocionais, socioeconômicas e culturais, uma vez que o contexto em que emerge a doença tem estreita relação com o cuidado que será estabelecido ${ }^{5}$. Nessa perspectiva, a prática interdisciplinar se mostra relevante para a construção do cuidado integral à criança e adolescente com câncer, pela vulnerabilidade desses grupos e pela necessidade de um cuidado de maior abrangência, dada a grande dependência e riscos iminentes ${ }^{6,7}$.

A partir disso, este estudo objetivou identificar as perspectivas de familiares de crianças e adolescentes com câncer quanto à assistência prestada pela equipe multiprofissional.

\section{MÉTODO}

Estudo descritivo, exploratório e com abordagem qualitativa, direcionado pelo protocolo internacional Consolidated criteria for reporting qualitative research (COREQ). 0 estudo foi realizado na Fundação Sara, localizada na cidade de 
Montes Claros, Minas Gerais (MG). Essa instituição foi escolhida em decorrência de ser a referência para o encaminhamento e tratamento de crianças e adolescentes com diagnóstico oncológico na região.

Foi enviada uma carta de apresentação e um Termo de Consentimento Institucional à direção clínica da instituição para autorização do estudo. A instituição foi devidamente orientada quanto às diretrizes da pesquisa, com assinatura de ciência autorizando a pesquisa. A coleta de dados foi realizada no segundo semestre de 2012, durante os meses de outubro e novembro, pelo pesquisador responsável.

A população do estudo foi constituída por 10 familiares de crianças e adolescentes com diagnóstico de câncer, em tratamento e acompanhamento oncológico, assistidos pelas instituições hospitalares de Montes Claros. Foram adotados os seguintes critérios de inclusão para participação no estudo: ter 18 anos ou mais; não apresentar limitações ou restrições fonoaudiológicas (deficiência auditiva e/ou vocal), tendo em vista a gravação oral dos depoimentos.

Para coleta de dados, utilizou-se entrevista semiestruturada, aplicando roteiro testado previamente com outros familiares de pacientes com perfis oncológicos semelhantes e contendo as seguintes questões norteadoras: (1) Quais são as dificuldades que você encontra diante do tratamento do seu filho? (2) Com relação ao tratamento do seu filho, quais cuidados são priorizados pelos profissionais de saúde? (3) Com o diagnóstico do câncer, várias mudanças são impactadas tanto na sua vida quanto na vida do seu filho. Quais mudanças são essas? (4) Quais são as formas de apoio e como elas são proporcionadas a você e ao seu filho por meio da equipe multiprofissional? (5) Como o enfermeiro, dentro da equipe multiprofissional, contribui para a prestação de cuidados para o seu filho? (6) Como é a interação da equipe multiprofissional com você e seu filho? (7) A equipe multiprofissional proporciona uma assistência que também contempla as suas necessidades? Quais delas são contempladas? (8) Quais são as necessidades que seu filho apresenta no tratamento do câncer? Em sua opinião, essas necessidades são supridas? (9) Os profissionais de saúde desempenham ações voltadas para o alívio da dor e conforto psicológico do seu filho? (10) 0 enfermeiro, no contexto da equipe 
multiprofissional, estabelece uma relação que te proporciona esclarecimento de dúvidas para a melhora da assistência do seu filho? (11) Você percebe que a equipe multiprofissional se envolve e se esforça o bastante para acompanhar você e seu filho em toda a trajetória da doença, desde os procedimentos e diagnósticos até a fase final desta?

Para a captação e armazenamento dos depoimentos, utilizou-se um celular com gravador MP3. 0 término da entrevista foi determinado por meio da saturação dos dados. Os depoimentos foram transcritos na íntegra, e analisados através da Análise de Conteúdo ${ }^{8}$, percorrendo três fases: (1) pré-análise, (2) exploração do material e (3) tratamento dos resultados, inferência e interpretação. Após análise das entrevistas, foram estabelecidas seis categorias para este estudo, sendo elas: "Preocupação médica na assistência prestada", "Assistência de enfermagem durante o tratamento", "Recebendo assistência e apoio psicológico", "O nutricionista participando do tratamento", e "Recorrendo à atuação do assistente social nos cuidados à família e ao paciente".
Os participantes foram devidamente orientados quanto às diretrizes do estudo e assinaram o Termo de Consentimento Livre e Esclarecido (TCLE) para participação voluntária da pesquisa. Foi garantido o sigilo e o anonimato dos participantes do estudo. Os depoimentos foram identificados por meio de códigos compostos pela letra "F", e números arábicos de 1 a 10 (F1-F10).

$\mathrm{O}$ estudo obedeceu aos preceitos éticos estabelecidos pela Resolução $\mathrm{n}^{\circ}$ $466 / 2012^{9}$, de 12 de dezembro de 2012, do Conselho Nacional de Saúde (CNS), com aprovação pelo Comitê de Ética em Pesquisa com Seres Humanos (CEP FIPMoc), Certificado de Apresentação para Apreciação Ética (CAAE) $n^{\circ}$ 07117312.0.0000.5109 e parecer consubstanciado $\mathrm{n}^{\circ} 130.035$.

\section{RESULTADOS E DISCUSSÃO}

\section{Preocupação médica na assistência prestada}

O sustento indispensável da relação médico-paciente, expresso pelos depoimentos foi a confiança. Foi possível também perceber o envolvimento deste profissional com 0 tratamento, 
esclarecimento de dúvidas, apoio e humanização.

O médico... fui muito bem tratada por ele, meu filho chegou desenganado [...]. Um médico me comoveu porque foi o único que disse que meu filho ia ficar uns trinta dias ali, mas ia ser reabilitado. $(\mathrm{F} 3)$

O médico sempre de 20 em 20 minutos passava lá para ver como é que ele estava. Dava toda a orientação e eu era bem recebida. ( $\mathrm{F} 4)$

O médico, assim o carinho, a atenção, a paciência. Quando eu perguntava tinha paciência para responder as dúvidas, né? (F5)

A médica deu muito apoio para minha menina, sempre deu força para nós. Até lá de casa eu ligo para médica e qualquer hora ela me atende para falar como minha filha tá. (F6)

O médico escuta a gente quando a gente pergunta alguma coisa, toda a hora ele passa no quarto para ver a situação do menino, explica sobre os medicamentos e as reações. (F7)

Estabelecer uma relação mais humanizada no processo de comunicação entre médico e paciente proporciona maior sensibilidade e confiança diante do sofrimento. Esse elemento favorece o vínculo, a expressão da intimidade do paciente e o diálogo aberto ${ }^{3,10}$. Cabe ainda ao profissional a superação de modelos de cuidados centrados na doença, baseados em tratamentos prescritivos, sem participação do paciente ${ }^{10}$. Estudo realizado no Rio de Janeiro (BR), menciona que o familiar ao buscar conhecer e entender o que se passa, precisa sentir segurança nos médicos e na equipe $^{3}$, e para isso, não somente aspectos cordiais devem ser inclusos na relação médico-paciente, mas o interesse em minimizar os sofrimentos manifestados e ocultos.

Ao serem questionados sobre a interação da equipe multiprofissional, observou aparentemente boa comunicação entre equipe, paciente e família. Destacaram também, a atenção por parte de médicos e enfermeiros na condução de uma assistência acolhedora.

Lá todo mundo tem uma boa comunicação com a gente, eles eram muito prestativos. (F2)

Moça, a comunicação era muito boa! Os médicos e enfermeiros até choravam junto comigo. Eles pegavam tanta amizade comigo e com meu filho, vinha cirurgião de outros setores comovido com meu menino, vinha oncologistas toda hora para ver o menino porque a situação dele era muito grave. A equipe do laboratório, o radiologista, o povo do tomógrafo, do raio- $x$, tem assim aquele envolvimento. (F3)

As pessoas de lá eram muito boas $e$ tratavam a gente muito bem, Graças a Deus! A gente foi muito bem recebida lá, comunicava muito bem com a gente, eu saí dali satisfeita. (F4)

Toda a equipe se preocupava muito comigo, porque minha filha todo mundo assim, tinha o maior carinho com ela. Ela é uma menina meiga, gostava muito das enfermeiras. Ela fazia desenho $e$ mostrava: aqui tia! (F5) 
Toda dúvida que a gente tinha a equipe de saúde sempre esclarecia, tinha uma boa comunicação pra atender a gente. (F6)

Eu percebi que todos têm aquela preocupação, tem todo aquele envolvimento pra cuidar dele 'direitim'. (F7)

Eu percebi que a equipe tinha toda aquela educação, conversava com nós, comunicava sobre tudo que tava acontecendo com ele, adulava ele. (F8)

Conforme os depoimentos, há um fluxo contínuo de interação e comunicação, principalmente em manter o paciente e família orientados sobre o tratamento, consultas e nos momentos de maior emoção, e que em ambas situações sejam pautadas na ética e empatia. Essa realidade do estudo também foi observada e ressaltada em pesquisa realizada em uma UTI adulto, apontando que assistir o paciente com câncer vai além de um check list a ser cumprido, envolve o acompanhamento de sua trajetória pessoal e familiar, desde os procedimentos diagnósticos até a terminalidade ${ }^{11}$.

\section{Assistência de enfermagem durante o tratamento}

Nos depoimentos, os familiares apontam que a enfermagem parece detalhar melhor os cuidados de rotina e essenciais, estando mais atenta à qualquer alteração nos padrões de normalidade. Assim como entre os médicos, houve também destaque as questões ligadas à comunicação.

Eles explicam tudo 'direitim' como é que dá o medicamento, antes eu não sabia, mas agora eu já sei. (F1)

A enfermeira falava a hora do remédio, como que era para tomar. Da minha parte o cuidado foi muito bom. (F2)

0 enfermeiro olhava tudo, enquanto não corria todo soro ele não saía de perto do menino, orientava que não podia comer comida de fora, comer bobagem, dava a medicação na hora certinha, ficava olhando, cuidavam na hora de pegar a veia para não perder. Só o enfermeiro que pegava a veia, só quando ele estava fazendo outra coisa que o técnico ia fazer. (F3)

O enfermeiro sempre ficava ao lado dele para saber da medicação, da alimentação, e sempre ficava ali olhando ele para quando ele sentisse alguma coisa, sempre ficava atento, eu sai dali satisfeita. (F4)

Quando eu ia lá e perguntava quando ia fazer a quimioterapia, o enfermeiro ia lá, quando eu dava uma cochiladinha, com o maior carinho me falar: tá na hora do medicamento! Então assim eles tinham muito carinho, muita atenção... As dúvidas que a gente tinha eles tiravam. Quando a bombinha apitava, eu ia lá e avisava, eles iam lá. A enfermeira, quando estava ocupada, ia $e$ pedia outro para ir lá. Todos tiveram muito carinho, nunca fui distratada, nunca deixou faltar nada. Então, assim, pra mim foi tudo bom. (F5)

Os enfermeiros, enquanto ela estava internada tinha a maior coisa com ela, $e$ minha filha até hoje gosta demais deles. 
Não faltou nada por parte da enfermagem, não tenho reclamação deles. Às vezes quando eu não aguentava ver minha filha quando tava vomitando sangue, aí eles vinham e me tirava de lá pra eu não ver aquilo, sempre dava apoio e força pra mim. (F6)

o enfermeiro explica todos os procedimentos que vai fazer até o que a gente tem que fazer quando chegar em casa, toda a orientação eles dão. Todos eles têm um carinho especial. (F7)

Como observado, a equipe de enfermagem se preocupava com um cuidado integral dos pacientes oncológicos atendidos. Trata-se de uma preocupação contínua com as orientações de cada procedimento realizado, bem como das informações à família, desdobrando para os cuidados intensivos e para aqueles que requeiram disciplina e rigor na realização.

A equipe de enfermagem tem papel indispensável no acolhimento e gerenciamento da assistência oncológica, pois possui competências únicas, em relação ao cuidado ${ }^{10,11}$. Estudo realizado na China, aponta que os enfermeiros são os profissionais que mais se preocupam com as necessidades familiares não atendidas, pois não concentram todas as atenções apenas na criança com câncer, no contexto clínico. Estes profissionais ajudam na promoção da adaptação à hospitalização oncológica, com ambientação favorável, atitudes mais calorosas e solidárias, além de atendimento competente e com informações adequadas ${ }^{12}$.

Embora a comunicação, empatia e os cuidados de rotina sejam valorizados pela família, é importante que a equipe de enfermagem consiga avançar para novos patamares. Um exemplo disso foi apontado em estudo no Reino Unido, onde há profissionais de enfermagem com prática avançada para adolescentes e jovens adultos (TYA ANP) que atuam em serviços de oncologia, abrangendo a prestação de cuidados diretos, educação, pesquisa e gerenciamento. Essa assistência de enfermagem é percebida como efetiva, especificamente em relação ao diagnóstico correto, prescrição segura e impacto positivo no tratamento ${ }^{13}$.

\section{Recebendo assistência e apoio psicológico}

Dialogar e apoiar o paciente sobre o momento que está sendo vivenciado é um dos objetivos do atendimento psicológico, e esses aspectos foram ressaltados pelos participantes do estudo.

Tinha a psicóloga que levava a criança para brincar, ia lá no quarto visitar ela. (F2) 
O psicólogo falava que qualquer coisa que a gente precisasse que não estava na nossa condição eu podia chegar para eles e pedir. (F4)

O psicólogo dava todo o apoio, brincava, entrava no quarto e conversava com a gente. Dizia que podíamos contar com ele. (F7)

O psicólogo também dava a gente muito apoio, que não é fácil nesse momento. (F8)

A presença contínua do psicólogo no quarto parece ter contribuído com a criança e seus familiares. Apesar de não ter sido mencionado pelos participantes, é comum o paciente oncológico sofrer com ansiedade, medo, desvalor, frustração, baixa autoestima, tristeza e depressão durante o tratamento, sendo fundamental a participação do psicólogo tanto em atendimento individuais como compartilhado com os demais membros da equipe, a fim de favorecer a ressignificação desta experiência ligada ao adoecer e sensação de morte ${ }^{14}$.

O psicólogo busca ferramentas que mobilizam recursos psíquicos infantis, por meio de brincadeiras, jogos e diálogo. Essas intervenções comumente são implementadas através da análise do processo de adoecimento ${ }^{14,15}$. A intervenção da psicologia durante o curso da doença contribui no processo de aceitação, reforço positivo e reabilitação.
Os depoimentos dos familiares corroboram com esta premissa, de modo a enfatizar o trabalho do psicólogo no apoio direto e a necessidades que o familiar não conseguia resolver sozinho.

\section{0 nutricionista participando do tratamento}

O nutricionista foi citado pelos familiares como orientadores e determinantes para uma alimentação adequada e específica para os pacientes em tratamento.

Tinha o nutricionista que passava no quarto e falava o que podia comer 'direitim'. (F2)

Meu filho tinha duas nutricionistas que prestava toda assistência com o alimento dele. $(\mathrm{F} 3)$

O nutricionista falava da alimentação, foi ótima alimentação para ele, o almoço era muito bom para ele, falava pra ele evitar coisa com muita gordura, com muito sal, carne de porco, até ovo. Falava para comer mais verdura, cenoura, beterraba, que é muito boa para o sangue. (F4)

O nutricionista dava toda orientação do que podia ou não comer, fazia o acompanhamento também porque no início, a dieta dela era livre. Qualquer coisa que ela bebia ou sentia mal a nutricionista ia e ligava para médica. (F5)

O nutricionista mesmo ficou bem de cima com a alimentação dele, porque assim ele tinha acabado de operar e aí então ele ficou muito tempo sem comer $e$ 
depois começou a alimentar só de liquido e assim, o nutricionista ficou o tempo todo em contato com ele e procurando o médico se já podia mudar a alimentação dele. (F7)

Observa-se a atenção ao acompanhamento nutricional diário, orientações ao paciente e a família, e a interação com outros profissionais da mesma categoria (outros nutricionistas responsáveis pela assistência) e com a equipe médica.

Os depoimentos enfatizam a presença do profissional nutricionista no acompanhamento do paciente, quanto ao consumo adequado de alimentos. Essa preocupação está relacionada às necessidades nutricionais para suportar o intenso tratamento, e apoiar o crescimento e desenvolvimento infantojuvenil durante e após a terapia antineoplásica. Estudo revela que pacientes com leucemia linfóide aguda (LLA) malnutridos, muito magros ou com sobrepeso, têm sobrevida menor do que os demais. Entretanto, com a correção do estado nutricional no decorrer do tratamento, novo prognóstico é estabelecido, mostrando que a participação do nutricionista na oncologia permite alterar um fator de risco modificável para essas crianças ${ }^{16}$.
O suporte nutricional pode ser utilizado como uma modalidade adjuvante na terapia oncológica. Sendo assim, uma boa terapia nutricional pode favorecer a dose máxima de administração quimioterápica, diminuir taxas de infecções, o tempo de permanência na internação e, assim, o custo hospitalar ${ }^{17}$.

\section{Recorrendo à atuação do assistente social nos cuidados à família e ao paciente}

O contato do assistente social com a família, não apenas no início do tratamento, permite a identificação de inúmeros aspectos e demandas como, situações socioeconômicas precárias; desavenças familiares; violência; falta ou problemas de moradia e alimentação; dificuldades de compreensão e de acesso a serviços de saúde, educação e assistência social. Ao tomar conhecimento dessas necessidades, o assistente social busca recursos na comunidade, a fim de garantir os direitos, acolhimento e apoio à família no enfrentamento da situação ${ }^{18}$.

Tem uma assistente social lá que essa é boazinha demais pra mim, resolve todos os papéis e manda pra secretaria da minha cidade. (F1) 
O assistente social preocupava e ajudava com algum documento. (F2)

$O$ assistente social me ajudava em tudo. (F3)

0 assistente social falava que qualquer coisa que a gente precisasse que não estava na nossa condição eu podia chegar pra eles e pedir. (F4)

A assistente social também ajudava. (F6)

Nos depoimentos, os familiares destacam que 0 assistente social se mostra disposto a resolver as questões burocráticas, como 0 envio de documentação e encaminhamentos. Em um estudo realizado em Centro Oncológico Pediátrico em Fortaleza (CE), as mães dos pacientes revelaram que os profissionais do Serviço Social se preocupavam integralmente com o bemestar das crianças, proporcionando companhia, ajuda em todos os âmbitos, administrativos e legais ${ }^{19}$. Outro estudo em Curitiba (PR) reforça esses achados, apontando que os cuidados a essas famílias necessitam de propostas e ações em vários níveis, articulando diversas políticas, na área de habitação, benefícios sociais, educação e assistência à saúde ${ }^{18}$.

A integração do assistente social dentro da equipe multiprofissional é necessária, pois possibilita para paciente e família o reencontro com seus direitos durante o tratamento e acesso a benefícios, como a presença integral de um acompanhante e revezamento de turno, transporte, realização de procedimentos e aquisição de medicamente sob custeio do Sistema Único de Saúde (SUS). Esses benefícios contribuem para a promoção do cuidado em saúde que não se restringe apenas ao diagnóstico médico, mas ao fator social e econômico que, por sua vez, interfere em toda a dinâmica do paciente oncológico e sua família ${ }^{20}$.

Esses profissionais juntamente com a equipe de enfermagem podem ainda enfrentar alguns desafios como, por exemplo, junto àqueles que além da condição oncológica, vivenciam outro transtorno que afeta o desenvolvimento do cérebro na infância e adolescência ${ }^{21}$, 0 que requer maior empenho da família e mobilização de recursos extras ${ }^{22}$. Nesse sentido, os profissionais normalmente buscam a construção de ambientes educativos e lúdicos dentro do hospital, com a inclusão de práticas não invasivas de alívio da dor ${ }^{23,24}$, a fim de minimizar os impactos da hospitalização, contribuir com o bem-estar do paciente e na sua colaboração durante o tratamento. 
0 estudo apresentou algumas limitações, principalmente em relação às características da coleta de dados e amostra, o que impede a generalização dos achados. Embora haja aumento gradual de trabalhos sobre a participação da equipe no cuidado oncológico, especificamente na área de enfermagem, outras áreas ainda carecem de estudos.

\section{CONSIDERAÇÕES FINAIS}

Os familiares vêem como importante o aumento da relação médicopaciente, a existência do vínculo com a equipe multiprofissional, a humanização nos cuidados prestados, a promoção do diálogo e na garantia dos direitos sociais. Apontaram ainda, que os cuidados à criança e adolescente com câncer devem ser compartilhados entre todos os envolvidos no processo de cuidado.

A vivência dessas famílias abrange várias dimensões do ser humano, que são diretamente afetadas pela condição de saúde de seus familiares em tratamento oncológico. Para o sucesso dessa assistência é importante o desenvolvimento do vínculo entre a equipe de saúde, o paciente e seus familiares, oportunidade que 0 profissional deve assumir postura ativa e assistir essa coletividade de forma integral e conforme suas particularidades. 0 atendimento aos anseios desses usuários, situando-os a um novo contexto de vida, ambientes e formas de cuidado auxilia no processo de adaptação, entendimento de suas necessidades e autocuidado.

\section{REFERÊNCIAS}

1. Teston EF, Fukumori EFC, Benedetti GMS, Spigolon DN, Costa MAR, Marcon SS. Sentimentos e dificuldades vivenciadas por pacientes oncológicos ao longo dos itinerários diagnóstico e terapêutico. Esc Anna Nery. 2018; 22(4):e20180017.

2. Saraiva DCA, Santos SS, Monteiro GTR. Tendência de mortalidade por leucemias em crianças e adolescentes nas capitais dos estados brasileiros: 1980-2015. Epidemiol Serv Saúde. 2018; 27(3):e2017310.

3. Afonso SBC, Minayo MCS. Relações entre oncohematopediatras, mães e crianças na comunicação de notícias difíceis. Ciênc Saúde Colet. 2017; 22(1):53-62.

4. França JRFS, Costa SFG, Lopes MEL, Nóbrega MML, Batista PSS, Oliveira RC. 
Existential experience of children with cancer under palliative care. Rev Bras Enferm. 2018; 71(suppl. 3):1320-7.

5. Castôr KS, Moura ECR, Pereira EC, Alves DC, Ribeiro TS, Leal PC. Palliative care: epidemiological profile with a biopsychosocial look on oncological patients. BrJP. 2019; 2(1):49-54.

6. Pacheco CL, Goldim JR. Percepções da equipe interdisciplinar sobre cuidados paliativos em oncologia pediátrica. Rev Bioét. 2019; 27(1):67-75.

7. Oliveira RAA, Zago MMF, Thorne SE. Interaction between professionals and cancer survivors in the context of Brazilian and Canadian care. Rev Latinoam enferm. 2017; 25:e2972.

8. Bardin L. Análise de conteúdo. Lisboa, Portugal: ed. 70; 2009.

9. Brasil. Ministério da Saúde. Conselho Nacional de Saúde. Resolução $n^{\circ}$ 466, de 12 de dezembro de 2012. Aprova as diretrizes e normas regulamentadoras da realização de pesquisa envolvendo seres humanos. Brasília: CNS; 2012.

10. Comin LT, Panka M, Beltrame V, Steffani JA, Bonamigo EL. Percepção de pacientes oncológicos sobre terminalidade de vida. Rev Bioét. 2017; 25(2):392-401.
11. Santos $D C L$, Silva $M M$, Moreira $M C$, Zepeda KGM, Gaspar RB. Planejamento da assistência ao paciente em cuidados paliativos na terapia intensiva oncológica. Acta Paul Enferm. 2017; 30(3):295-300.

12. Liy QY, Wong FKY, You LM, Zhou XZ. Unmet Family Needs Concerning Healthcare Services in the Setting of Childhood Hospitalization for Cancer Treatment in Mainland China: A Qualitative Study. J Pediatric Nurs. 2019; 44:e66-e71.

13. Masters E, Weston C, Chisholm J, Soanes L. Role of the Advanced Nurse Practitioner Within Teenage and Young Adult Oncology. What is the Impact on Patient and Staff Experience of a New Nurse Practitioner Role to a Teenage and Young Adult Service? J adolesc young adult oncol. 2019; 8(6):668-673.

14. Alves SWE, Uchôa-Figueiredo LR. Estratégias de atuação da psicologia diante do câncer infantil: uma revisão integrativa. Rev SBPH. 2017; 20(1):5574.

15. Silva PLN, Xavier GC, Oliveira VV, Figueredo ML, Prado PF, Aguiar Filho W. Câncer infantil: vivências de 
crianças em tratamento oncológico. Enferm Foco. 2016; 7(3/4):51-5.

16. Viani K, Albuquerque L, Barr RD, Ladas EL. Nutrition of children with cancer in Brazil: a systematic review. JCO Glob Oncol. 2020; 1(6):242-59.

17. Viani K, Barr RD, Odono Fllho D, Ladas EL. Nutritional status at diagnosis among children with cancer referred to a nutritional service in Brazil. Hematol Transfus Cell Ther. 2020; 30(20):1-7.

18. Xavier PRH, Lopes SMB. As práticas do assistente social e sua articulação com a equipe de saúde de um hospital de Curitiba. Serv Soc Rev. 2016; 18(2):132-50.

19. Santos EC, Vasconcelos APSM. A atuação das assistentes sociais vista pelas famílias das crianças atendidas em um Centro Pediátrico do Câncer. Serv Soc Saúde. 2015; 14(2):261-78.

20. Silva TP, Leite JL, Stinson J, Lalloo C, Silva IR, Jibb L. Estratégias de ação e interação para o cuidado à criança hospitalizada com dor oncológica crônica. Texto Contexto - Enferm. 2018; 27(4):e3990017.

21. Moreira MTF, Lima AMN, Guerra M. Sobrecarga do cuidador informal de crianças com transtorno do espectro do autista. J Health NPEPS. 2020; 5(1):38-51.

22. Torres-Reyes A, Tenahua-Quitl I, García-López MA, Pérez-Noriega $E$, Cordero-Sánchez C, Ramos-Durán N, et al. Relación de calidad de vida y autocuidado en pacientes adultos con cáncer. J Health NPEPS. 2019; 4(1):1630.

23. Osorio-Sandoval JA, Torres-Reyes A, Tenahua-Quitl I, De-Avila-Arroyo ML, Morales-Castillo FA, García-Lopez MA, et al. Efectividad de la risaterapia en manejo el dolor en pacientes pediátricos postoperados de apendicetomía. J Health NPEPS. 2019; 4(2):44-57.

24. Mendes DS, Moraes FS, Lima GO, Silva PR, Cunha TA, Crossetti MGO, et al. Benefícios das práticas integrativas e complementares no cuidado de enfermagem. J Health NPEPS. 2019; 4(1):302-318. 
Financiamento: Os autores declaram que não houve financiamento.

Conflito de interesses: Os autores declaram não haver conflito de interesses.

\section{Participação dos autores:}

- Concepção: Silva PLN, Martins FGS, Freire JD, Miranda FB, Souza AAM.

- Desenvolvimento: Silva PLN, Martins FGS, Freire JD, Miranda FB, Souza AAM.

- Redação e revisão: Silva PLN, Martins FGS, Freire JD, Miranda FB, Souza AAM.

Como citar este artigo: Silva PLN, Martins FGS, Freire JD, Miranda FB, Souza AAM. Perspectivas de familiares de crianças e adolescentes em tratamento oncológico quanto à assistência multiprofissional. J Health NPEPS. 2020; 5(2):60-74.

Submissão: $19 / 02 / 2020$

Aceito: $28 / 07 / 2020$

Publicado: 04/12/2020 